\title{
Promoción de la salud a través del arte: estudio de caso de un taller de fotografía en "Ciudad Oculta", la villa $N^{\circ} 15$ de la Ciudad de Buenos Aires
}

\author{
Community arts and health promotion: a case study in a \\ photography workshop in "Ciudad Oculta" (Hidden \\ City), the shanty town $\mathrm{N}^{0} 15$ of the City of Buenos Aires
}

Gabriela Wald ${ }^{1}$

\footnotetext{
${ }^{1}$ Magister en Educación, Promoción de la Salud y Desarrollo Internacional, Institute of Education, University of London. Estudiante de Doctorado en Ciencias Sociales, Universidad de Buenos Aires, Argentina. gawald@gmail.com
}

RESUMEN El presente trabajo analiza las potencialidades del arte comunitario para promover la salud y el bienestar entre jóvenes que viven en contextos de vulnerabilidad social en América Latina. A través de un estudio de caso se propone aportar elementos para el debate teórico y metodológico de una problemática central en promoción de la salud: la distancia entre su retórica, basada en una concepción de salud amplia -entendida como proceso colectivo e integral, influido por aspectos sociales, económicos, ambientales y culturales- y la mayor parte de las prácticas que bajo esta filosofía se han realizado en nuestra región, intervenciones de tipo preventivo organizadas alrededor de conceptos de enfermedad y que responden a modelos teóricos individualistas. El caso seleccionado es "ph15", una experiencia de educación fotográfica gestionada por un grupo de fotógrafos en "Ciudad Oculta", la villa No 15 de la Ciudad de Buenos Aires. La investigación muestra que los jóvenes perciben cambios individuales y colectivos como consecuencia de participar en este taller, cambios que pueden vincularse al concepto de salud en sentido amplio. Así, una experiencia que no fue pensada para promover la salud puede aportar elementos para diseñar intervenciones más acordes a la filosofía en promoción de la salud.

PALABRAS CLAVE Promoción de la Salud; Fotografía; Áreas de Pobreza; Adolescente; Argentina.

\footnotetext{
ABSTRACT This article analyzes the potentials of community art to promote health and wellbeing among young people living in vulnerable social contexts in Latin America. Through a case-study, we aim at providing elements for the theoretical and methodological debate of a central problem in health promotion: the distance between its rhetoric, based on a wide conception of health -understood as a collective process, influenced by social, economic, environmental and cultural issues- and the interventions that have been carried out in our region, organized around disease concepts that respond to individualistic theoretical approaches. The selected case is ph15, a photography workshop delivered by a group of photographers in the shanty town $N^{0} 15$ "Ciudad Oculta" ("Hidden City") of the City of Buenos Aires. The study shows that young people notice individual and collective changes as a result of participating in this workshop, and these changes could be linked to the wide concept of health. Therefore, an activity that has not been thought to promote health can help us to design activities that are closer to the philosophy of health promotion.
}

KEY WORDS Health Promotion; Photography; Poverty Areas; Adolescent; Argentina. 


\section{INTRODUCCIÓN}

El presente trabajo analiza las potencialidades del arte comunitario para promover la salud y el bienestar entre jóvenes que viven en contextos de vulnerabilidad social y pobreza estructural en un país de medianos ingresos como la Argentina. Se propone aportar elementos para discutir uno de los problemas centrales del campo de la promoción de la salud: la distancia entre una retórica aceptada en el mundo occidental, basada en una concepción de salud amplia (1), entendida como proceso colectivo e integral, influido por aspectos sociales, económicos, ambientales y culturales (2-5) y las prácticas que bajo su nombre se llevan a cabo.

Las intervenciones en promoción de la salud han sido variadas en cada uno de los países, así como al interior de los mismos. En Gran Bretaña y Canadá, por ejemplo, los gobiernos se apropiaron del concepto holístico de salud y algunos abordajes en promoción de la salud fueron modificados. Así, en esos países se desarroIlaron intervenciones sociopolíticas multisectoriales que trascendieron el sector salud $(2,6)$.

Sin embargo, en América Latina los programas y proyectos en promoción de la salud no han logrado poner en práctica intervenciones innovadoras, razón por la cual algunos analistas consideran que la promoción de la salud en nuestra región es más bien retórica vacía $(7,8)$. El problema central es que a pesar de definirse la salud en términos de bienestar, las prácticas continúan organizándose alrededor de conceptos de enfermedad (9). Así, la mayoría de los programas implementados en promoción de la salud son en realidad intervenciones de tipo preventivo y responden, en última instancia, a modelos teóricos de base individualista y conductista $(5,10)$. Además, no se han hecho extensivos los declamados abordajes intersectoriales y transdisciplinarios; por lo tanto, los programas de promoción de la salud son gestionados solo por el sector salud (8).

A estas consideraciones es preciso agregar que en contextos de vulnerabilidad social, pobreza estructural y violencia -como es el caso de muchas ciudades de nuestro país- los enfoques de promoción de la salud gestionados desde el sector salud que se proponen la adopción de pautas de cuidado se exponen a una serie de contradicciones: ¿cómo priorizar, por ejemplo, la visita al centro de salud cuando hay urgencias que atender, como ser cuestiones de comida, vivienda e ingresos? Si se entiende a la salud como un proceso integral y se reconoce que los determinantes de salud son múltiples, que no pueden ser abordados solo desde el sector salud, ¿por qué las intervenciones continúan ligadas al fortalecimiento de la atención primaria de la salud y al cambio de comportamiento? ¿Será que nuestros gestores confunden Atención Primaria de la Salud con promoción de la salud? ¿Por qué no trabajar en programas integrales que impliquen mejoras en diversos aspectos de la vida de las personas, aspectos que puedan vincularse con el bienestar tanto a nivel personal como colectivo? Estas preguntas, enfatizo, son centrales cuando se piensa la promoción de la salud en países con altos índices de pobreza e inequidad.

Si hablamos de la población joven estas contradicciones se suman a las consecuencias que los cambios estructurales y sociales en nuestro país han provocado en ellos. Según Urresti (11) es posible observar una merma en las bases estructurales de la participación en general, y de la participación juvenil en particular. En palabras de Claudia Jacinto y Carla Bessega (12) los jóvenes pobres son uno de los grupos más vulnerables de nuestra sociedad "al ser los más afectados por la escasez y precarización del empleo, y por la fragilización de los vínculos sociales". Teniendo en cuenta estos diagnósiticos, ¿cómo lograr la participación de jóvenes de sectores populares en programas de promoción de la salud que trabajan contenidos específicos de salud en un contexto de "desencanto juvenil frente a las instituciones"? (13).

Este artículo se propone aportar elementos para el debate teórico y metodológico en promoción de la salud en particular y en intervenciones integrales en contextos de vulnerabilidad social y pobreza estructural en general. Se analizarán, a partir de un estudio de caso, las potencialidades del arte comunitario para producir cambios en ciertos aspectos de la vida cotidiana de los participantes, así como en sus propias representaciones sociales, de ellos mismos, del lugar en el que viven y de otros sectores sociales. La selección de este caso estuvo inspirada en la 
creciente visualización de un abanico de experiencias de arte comunitario en barrios o villas, primero en Buenos Aires (área metropolitana) y luego en el interior del país. Durante los últimos diez años se desarrollaron y se hicieron visibles diversos talleres de teatro, cine, danza, fotografía, música (popular, orquestal), etc., en su mayoría dirigidos a jóvenes cuyos productos artísticos lograron atravesar las barreras barriales y ser exhibidos en galerías y centros culturales frecuentados por sectores sociales medios y medios altos. No ha habido hasta el momento ningún relevamiento formal de estas iniciativas, a pesar de aparecer con frecuencia en los medios de comunicación masivos y de ser cada vez más conocidas por la sociedad argentina.

Uno de los proyectos que ha persistido durante más de ocho años y ha exhibido sus productos en Argentina y el extranjero es el taller de fotografía "ph15" que se dicta en "Ciudad Oculta", la villa No 15 de la Ciudad de Buenos Aires. El proyecto comenzó en el año 2000 cuando el fotógrafo Martín Rosenthal -a pedido de algunos jóvenes del barrio- inauguró un espacio de enseñanza fotográfica para jóvenes. Ese primer espacio creció y se estableció con la participación de Moira Rubio y Miriam Priotti, transformándose en un taller y fundación que en 2005 -año en que se realizó el trabajo de campo- involucraba a 26 adolescentes y jóvenes entre 13 y 25 años. En 2006, ph15 consiguió financiación regular de una fundación extranjera y replicaron el proyecto en otros dos barrios, esta vez del conurbano bonaerense. Los aproximadamente 75 participantes del proyecto producen alrededor de 60.000 fotos al año y exhiben de manera periódica su trabajo en centros culturales y museos de Buenos Aires, el conurbano, el interior del país y su obra ha viajado también al extranjero (España y EE.UU.).

El presente artículo analiza la experiencia de ph15 desde la perspectiva de los participantes, sus docentes y algunos padres. Se describirá, en primer lugar, si identifican cambios como consecuencia de la participación de los jóvenes en ph15 y cuáles son estos cambios. Se indagará también si los mismos se perciben como individuales y/o colectivos, y cómo ocurren. Finalmente, se discutirán los límites y potencialidades de ph15 en particular, y de experiencias de arte comunitario en general, para ser pensadas como estrategias de promoción de la salud integral, fundamentalmente en contextos de vulnerabilidad social y pobreza estructural.

\section{METODOLOGÍA}

La estrategia metodológica utilizada ha sido cualitativa: se realizó un estudio de caso del taller de fotografía ph15. El estudio triangula información obtenida a través de cinco meses de observación participante, 19 entrevistas en profundidad (12 con jóvenes del taller, cuatro con docentes y tres con madres) y análisis de documentos (el sitio web de ph15 y otros materiales de promoción de actividades propias, algunos artículos en diarios y un libro editado por ph15 con fotografías de los jóvenes). En todas las etapas se tuvieron en cuenta medidas para la protección de la confidencialidad de la información y el anonimato de los informantes. En todas las entrevistas se realizó el proceso de consentimiento informado que constó de dos etapas: una conversación para informar los objetivos de la investigación y el uso que se dará a sus testimonios, pudiendo allí los potenciales entrevistados hacer preguntas sobre aquello que no hubiera quedado claro; y un momento en el cual se firmó dicho consentimiento.

El análisis se realizó con apoyo del software Atlas Ti. Se utilizaron tanto categorías definidas por la literatura, previas al análisis de los datos, como categorías y códigos emergentes de las entrevistas, observaciones y documentos. Una vez codificados los datos, los tres grupos de entrevistas (jóvenes, madres y docentes) fueron analizados en dos sentidos: como si fueran un corpus único, identificando temas, patrones, relaciones y percepciones comunes, y cada grupo por separado, para identificar tendencias, contradicciones y diferencias entre los grupos y al interior de cada grupo.

\section{CARACTERÍSTICAS DE LA MUESTRA}

De los 26 adolescentes y jóvenes que participaban en ph15 cuando se realizó el trabajo de campo varios son hermanos y primos. Así, 
si bien fueron entrevistados 12 jóvenes, la muestra involucra a nueve hogares. El Cuadro 1 describe las edades, el sexo y el tiempo que los entrevistados llevan concurriendo al taller.

Si bien la mayoría de los participantes del ph15 aún está en edad escolar (hasta 18 años), tres de ellos abandonaron la escuela en 2005, cinco han repetido alguna vez un año escolar y solo uno de los adolescentes en edad escolar no ha repetido ningún año. Las familias de los participantes de ph15 también presentan bajos índices de escolaridad: ninguno de los padres finalizó el colegio secundario, si bien al momento de esta investigación dos de ellos se encontraban participando en programas de secundario para adultos. En estos hogares, ningún hermano había finalizado el colegio secundario.

Con relación a la composición de los hogares, solo en uno de los nueve hogares de esta muestra el padre y la madre aún conviven. Los tres hijos de esta familia que participan en ph15 -del grupo de graduados- son los únicos de toda la muestra que no abandonaron la escuela ni repitieron años. Los otros nueve entrevistados comentaron haber sido criados en hogares con diversos grados de inestabilidad (económica, familiar). Algunos de los padres de estos nueve jóvenes han sido usuarios de drogas y alcohol -con consumos problemáticos-; otros han ido o están en la cárcel; en otros casos el padre o la madre los abandonó cuando eran niños; en un caso el padre golpeaba a la madre, hasta que fue expulsado de la casa y encarcelado en 2003. En este contexto de inestabilidad en los vínculos afectivos, una participante mencionó haber sido abusada sexualmente por un pariente; otra haber vivido en un hogar para niños con algunos de sus hermanos, porque tanto su padre como su madre eran alcohólicos; y otro abandonó la casa de su madre para irse a vivir con su hermana mayor porque sus tres hermanos mayores, todos consumidores de drogas, vendían o empeñaban todos los objetos de la casa para obtener dinero, incluso la ropa de él.

Los datos y situaciones expuestos hasta aquí permiten aproximarse al contexto familiar que experimentan los jóvenes participantes de ph15. Sin embargo, para una contextualización más completa es preciso abordar qué construcciones realizan estos jóvenes de su barrio y cómo enmarcan su experiencia cotidiana en ellas.

\begin{tabular}{llll} 
Cuadro 1. GRUPOS DE TRABAJO DEL PROGRAMA PH15, SEGÚN EDAD, SEXO Y \\
TIEMPO EN EL PROYECTO. CIUDAD OCULTA, ARGENTINA. \\
\hline GRUPOS DE TRABAJO & EDAD & SEXO & TIEMPO EN PH15 \\
\hline "LOS MÁS CHICOS" & 14 & Varón & 1 año \\
& 14 & Mujer & 6 meses \\
& 14 & Mujer & 11 meses \\
& 16 & Varón & 1 año \\
"LOS GRANDES" & 16 & Mujer & 6 meses* \\
& 17 & Varón & 6 meses \\
& 17 & Mujer & 13 meses \\
"LOS GRADUADOS" & 17 & Varón & 3 años \\
& 18 & Mujer & 3 años y 6 meses \\
& 20 & Varón & 3 años \\
& 25 & Mujer & 3 años \\
& & Varón & 3 años
\end{tabular}

Fuente: Elaboración propia. 


\section{CIUDAD OCULTA Y LA EXPERIENCIA DEL BARRIO}

-"...hay $50 \%$ de posibilidad de que me peguen un tiro, aparte... no solamente que me peguen un tiro de lleno a mí, o sea, vos estás jugando a la pelota ahí adentro del barrio y se agarran a los tiro y vos estás en el medio, y tengo... tres amigos que ya les pasó eso, se comieron un tiro por cosas de otros."

- ¿Y por qué decís que tenés $50 \%$ de probabilidades de que te peguen un tiro?"

-"Y, porque todo el tiempo están tirando..." (Participante varón, 17 años)

Ciudad Oculta ha sido considerada una de las villas más peligrosas de la Ciudad de Buenos Aires. Los medios de comunicación -y por consiguiente la opinión pública- han construido una imagen de Ciudad Oculta ligada al crimen, al tráfico y uso de drogas y a la violencia cotidiana. Con aproximadamente 10.000 habitantes, es la sexta villa en tamaño de las 23 que hay en la Ciudad (14).

Las Figuras 1 y 2, ilustran los modos de mirar el propio barrio -y de contarlo- que tienen los jóvenes participantes de ph15. Es posible observar en ellas tanto el relato de los problemas de infraestructura a los que se enfrentan cotidianamente como cierta estilización de los mismos. Para contextualizar el análisis que propone este trabajo, es importante comenzar con las siguientes preguntas: ¿Qué piensan los jóvenes, sus padres y docentes sobre el barrio y la vida dentro de él? ¿Coinciden con la mirada que los medios de comunicación y otros sectores sociales tienen de sus espacios de sociabilidad?

En primer lugar, vale la pena aclarar que exceptuando una de las familias de los jóvenes entrevistados, el resto de los padres y hermanos nacieron o fueron criados en Ciudad Oculta. Por eso fue posible encontrar en todas las entrevistas un sentimiento de ambigüedad con respecto a la vida cotidiana en el barrio.

Por un lado, la mayoría de los entrevistados reconoce que en Ciudad Oculta todos se conocen con todos desde que eran chicos, y que eso genera una sensación muy fuerte de familiaridad. Sin embargo, dicen que la villa hoy no es lo que era y todos los entrevistados señalaron que los tiroteos y el uso de drogas son hoy moneda corriente. Así, un barrio que era percibido como familiar y tranquilo se transformó para ellos en un espacio de tensión casi permanente.

Todos los entrevistados comentaron que la población del barrio, especialmente la población joven, está dividida en tres, según la zona del barrio en la que viven. El primer grupo está compuesto por los que viven en "la villa", "el frente" o "la 15", al noreste del barrio. Aquí las casas están construidas en pasillos irregulares, bastante cerca unas de las otras y la instalación sanitaria y eléctrica es precaria.

El segundo grupo lo forman aquellos que viven en el "barrio nuevo", un sector construido bajo planificación estatal en el noroeste de la villa. Aquí las casas tienen electricidad y agua corriente, en un sector con pasillos más abiertos. En esta zona del barrio hay un enorme edificio abandonado ocupado por familias sin techo. A la tercera zona de la villa la llaman informalmente "el fondo" o "la 30", y es la zona más precaria, hacia el sureste. Hay casas hechas de material pero también de chapas, maderas y hasta cartones. Los participantes de ph15, que viven en "la 15 " y "en el barrio nuevo" dicen que en esta zona viven "los paraguayos".

Muchos entrevistados cuentan que los grupos de jóvenes de las tres zonas confrontan entre ellos casi todos los días, muchas veces con armas de fuego. Algunos mencionaron que la gente del "barrio nuevo" roba en "la villa" y viceversa, y que la gente "del fondo" viene al "frente" a robar. Además, casi todos los jóvenes y madres entrevistados mencionaron que hay tiroteos casi todos los días, elevando la posibilidad de caer en alguno sin siquiera participar en las peleas. Muchos también mencionaron que aquellos vecinos que se dedican al delito comenzaron a robar adentro de Ciudad Oculta. De hecho, dos de los jóvenes entrevistados contaron que fueron asaltados por sus propios vecinos.

La mayoría también mencionó que hay aspectos culturales que contribuyen al crecimiento de la confrontación entre vecinos. Dicen que, para ser respetados en Ciudad Oculta, la gente -especialmente los varones- tienen que mostrar que son fuertes, duros, que pueden soportar el dolor físico y que no tienen miedo de pelear con 
Figura 1. FOTOGRAFÍA DE CIUDAD OCULTA TOMADA POR NATALIA GODOY. PROYECTO PH15. CIUDAD OCULTA, ARGENTINA

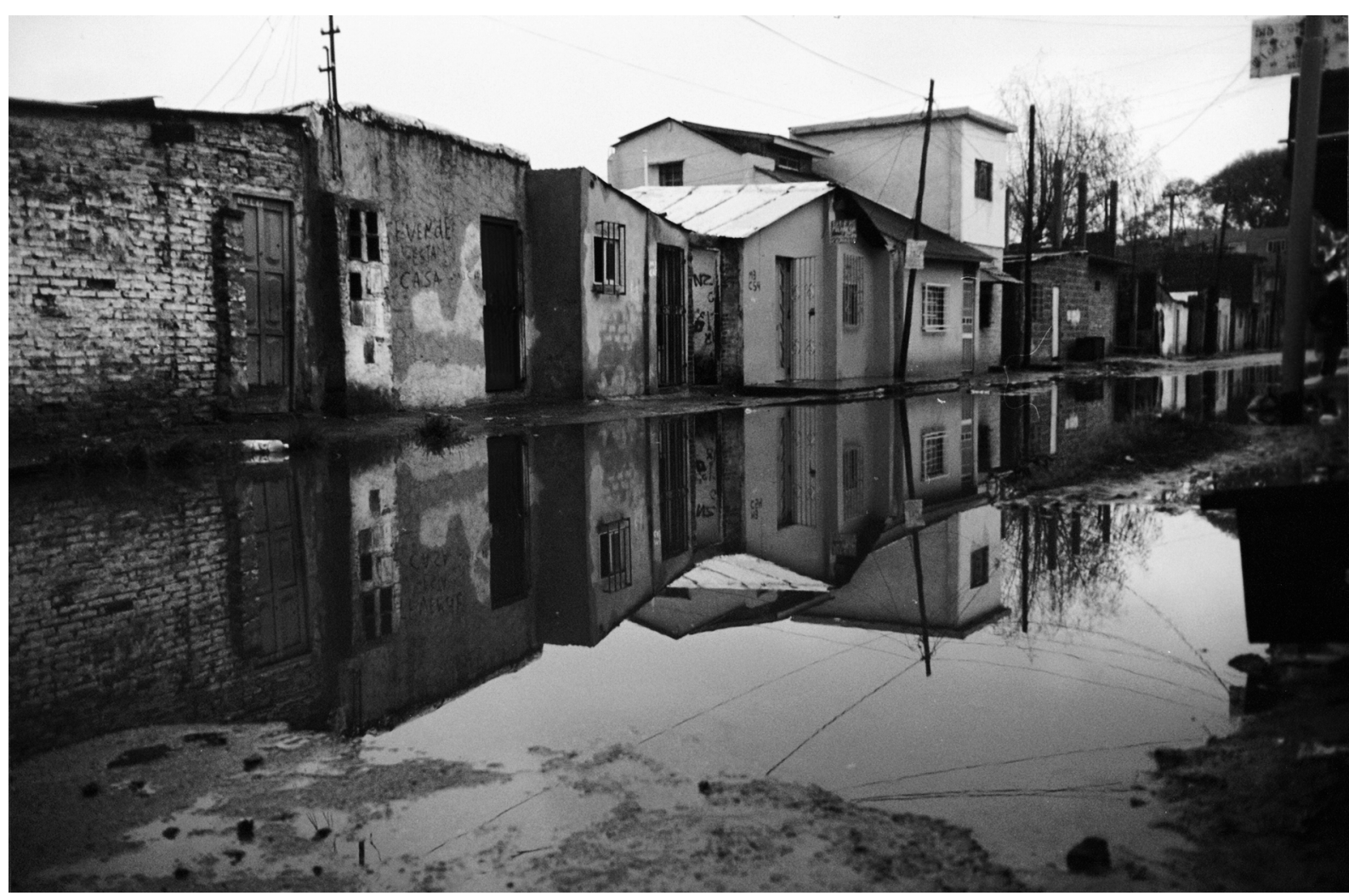

Nota: reproducido con la autorización de los autores y del Proyecto ph15@.

su propio cuerpo si es necesario hacerlo. Así, todo aquel que no adhiera a este modelo de hombre fuerte y resistente corre mayores riesgos de ser provocado y, eventualmente, golpeado o atacado. Algunos afirman también que incluso las mujeres prefieren salir o armar pareja con hombres que se identifican como los más fuertes para sentirse protegidas. En este contexto también el uso y el tráfico de drogas aparecieron como otras de las causas de la violencia, particularmente de los tiroteos.

Sin embargo, a pesar de que Ciudad Oculta es percibida como un ámbito violento y confrontativo por la gran mayoría de los entrevistados, para ellos su barrio tiene muchas cosas positivas: conocerse entre vecinos desde hace muchos años genera que la circulación entre los hogares sea permanente: las casas están siempre abiertas para recibir amigos y vecinos, para ofrecer un mate, comida o algún elemento que falte. Muchos jóvenes dijeron también no estar de acuerdo con el modo en que los medios mues- tran el barrio: retratan lo más precario, "lo más pobre y lo más decadente". Y si bien ellos reconocen las dificultades de su propio barrio, estos jóvenes creen que hay mucha gente honesta y trabajadora que los medios, y a través de ellos el público en general, no logran ver.

La ambigüedad que sienten los jóvenes y las madres entrevistadas en relación a Ciudad Oculta se expresa en que, por un lado, la mayoría dijo que le gustaría mudarse y vivir en otro barrio si pudieran. Sin embargo, a la vez, varios de los que eventualmente querrían irse dijeron que les sería difícil en un plano afectivo, porque tienen sus amigos y sus familias, y porque se sienten a gusto con la mayoría de los vecinos del barrio.

Estas percepciones, opiniones y reivindicaciones que tienen los jóvenes sobre el lugar en el que viven tienen tres implicancias. En primer lugar, son centrales para contextualizar la experiencia de educación fotográfica que este trabajo analiza. En segundo lugar, permiten comprender las diferencias de percepción entre los 
ámbitos de socialización cotidianos de los jóvenes y el espacio de trabajo en ph15. Finalmente, permiten enmarcar los resultados de este estudio en los esquemas de interpretación y sentido que los jóvenes construyen en relación al lugar que ocupan, y el que les gustaría ocupar, en el espacio social.

\section{PH15: EL PROYECTO Y LA PROPUESTA PEDAGÓGICA}

\footnotetext{
...nos dio una cámara y nos dijo "vayan a sacar" y yo pensé que me iban a decir, "bueno, tienen que sacar esto", pero no, me dijo "bueno, saquen" y, ¿cómo saquen?, pensé yo, y viste, lo miré como así diciéndole, ¿qué saco? Y me dijo, "lo que te guste, jlo que te Ilame la atención!" (Participante mujer, 18 años)
}

Ph15 comenzó en el año 2000 como una iniciativa independiente del fotógrafo Martín Rosenthal en respuesta a un pedido de jóvenes del barrio. El taller nació como una iniciativa espontánea para la enseñanza de fotografía, no como un proyecto para mejorar indicadores socioculturales entre jóvenes que viven en contextos de vulnerabilidad social. Por eso, se desarolló sin ningún tipo de financiamiento. En el año 2002, Moira Rubio se unió al proyecto como codirectora y docente. Juntos sostuvieron el taller y crearon la Fundación ph15 para darle un marco formal al proyecto. Entre los años 2005 y 2006 se realizó el trabajo de campo para este estudio. En aquel momento ph15 lo conformaban cinco docentes y 26 jóvenes de Ciudad Oculta. Dado que el taller había nacido de manera espontánea, el propósito y los objetivos de ph15 no estaban escritos en ningún documento fundacional, no había misión ni resultados esperados y las mejoras

Figura 2. FOTOGRAFÍA DE CIUDAD OCULTA TOMADA POR NANCI ALFONSO. PROYECTO PH15. CIUDAD OCULTA, ARGENTINA

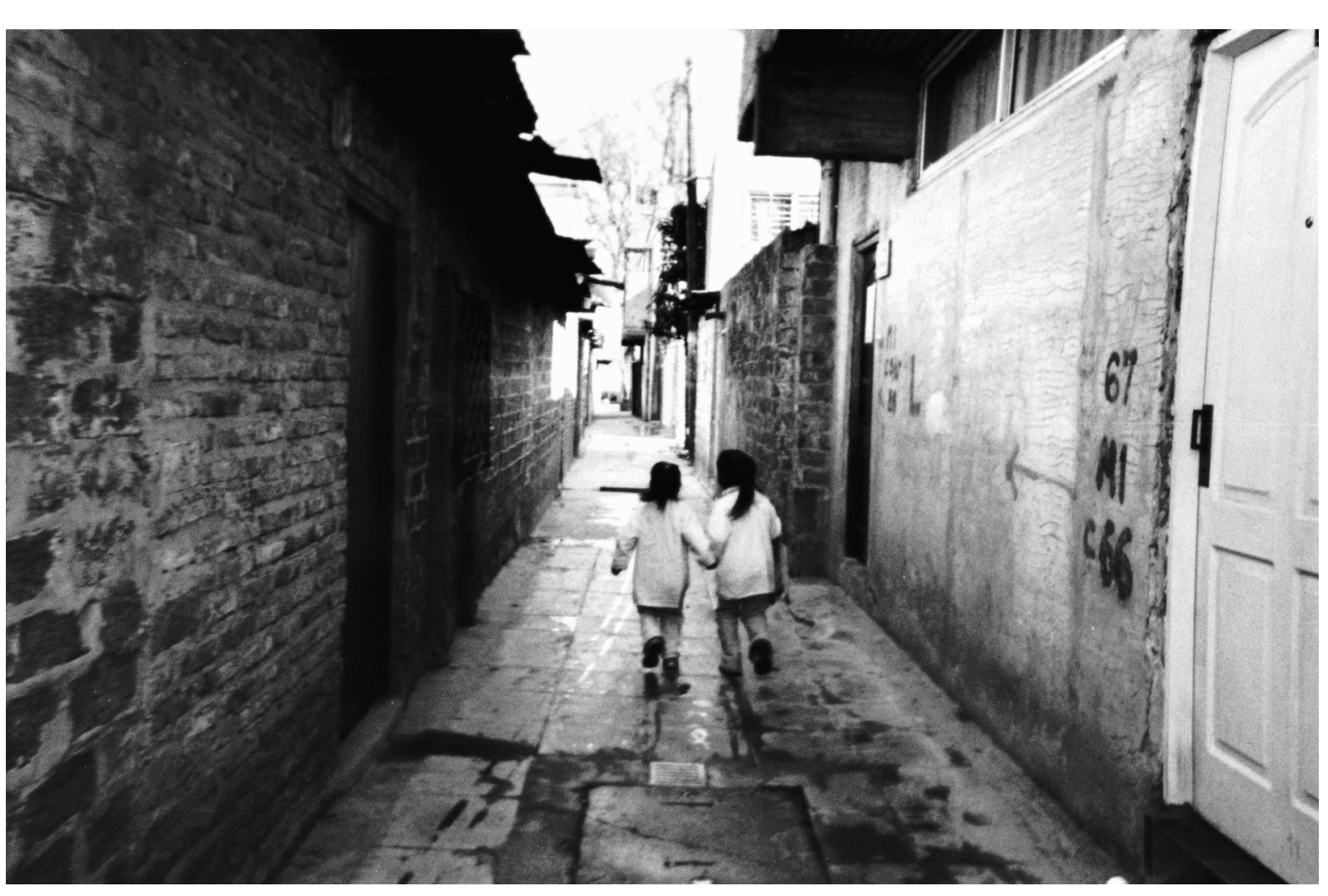

Nota: reproducido con la autorización de los autores y del Proyecto ph15@. 
en la intervención eran consecuencia de la reflexión conjunta de los docentes, derivada de la propia práctica.

Hasta fines del año 2006 ph15 no recibía ninguna financiación regular y se mantenía mayormente con donaciones de empresas o particulares que lograban conseguir de manera intermitente. Además, las fotografías de los participantes estaban (y están) a la venta, y son adquiridas -aunque no con mucha frecuencia- por personas en Argentina y el extranjero, principalmente en EE.UU. Cuando se vende una fotografía, el 50\% del valor lo recibe el o la autor/a y el otro $50 \%$ se lo queda ph15 para sostener el taller. Tan inestable fue el financiamiento de ph15 durante sus primeros siete años de vida que los directores confesaron haberse hecho cargo ellos mismos de la compra de materiales y revelados para que el taller pudiera seguir funcionando (a).

Los docentes describen la metodología de aprendizaje del taller como flexible y personalizada. Dicen que su objetivo es desmitificar la percepción de los jóvenes de que la fotografía es una disciplina compleja. Para enseñarla dividieron a los jóvenes en tres grupos: "los más chicos" (aprox. 10 adolescentes de 12 a 16 años), "los grandes" (otros 10 jóvenes de 17 a 20 años) y "los graduados", aquellos jóvenes que han estado en ph15 por más de tres años.

Cuando un nuevo participante se une al proyecto, ella o él, recibe, en el primer encuentro al que asiste, una cámara pocket y un rollo de fotos blanco y negro. Sin ninguna instrucción técnica (salvo el uso del flash) los docentes le proponen salir a sacar fotos de aquello que los jóvenes quieran, lo que se les ocurra, lo que vean en su vida cotidiana. Por eso, los alientan a llevar siempre su cámara de fotos con ellos, vayan adonde vayan.

Los participantes sacan fotografías durante la semana y los sábados se reúnen en una clase de análisis y crítica fotográfica. Los docentes revelan los rollos, hacen tiras de contactos y copian todas aquellas fotografías que consideran que pueden servir para enseñar la disciplina. Esta es la principal metodología: aprender de los propios errores. Los docentes trabajan aquí cuestiones de encuadre y composición de imágenes. Además, intentan que los jóvenes conecten sus fotografías con aquello que sentían o pensaban al momento de tomarlas, les preguntan cuáles fueron las razones para un encuadre determinado, cómo y cuándo lo hicieron, qué les transmitía aquello que estaban retratando, etc.

De la observación y las entrevistas fue posible inferir que los docentes de ph15 consideran a la fotografía una disciplina artística para la expresión de sentimientos y emociones, es decir, subyace una concepción esencialista del arte. Los cuatro docentes entrevistados han definido ph15 como un "taller expresivo" en el cual los jóvenes pueden aprender un lenguaje nuevo, una herramienta para la expresión personal. Así, consideran que cada joven tiene razones -más o menos inconscientes- para seleccionar aquello que fotografía. Piensan también que su función como docentes es ayudar a los jóvenes a encontrar estas razones y enseñarles a expresarlas y compartirlas con los demás.

Además de estos encuentros semanales hay tres tipos de actividades más que el taller promueve. La primera es visitar museos, galerías y centros culturales para ver exposiciones, en su mayoría de fotografía. Es importante aclarar que ninguno de los participantes había visitado una exhibición artística antes de unirse a ph15. Cuando los expositores son argentinos, los docentes tratan de contactarlos para que estén presentes el día que los jóvenes irán de visita. Esto permite a los participantes tener contacto directo con los artistas y, además de ver su obra, conversar sobre la misma, sobre cómo la hicieron y con qué ideas trabajaron. La segunda actividad que ph15 promueve es la visita de otros docentes o fotógrafos al taller, para realizar actividades especiales durante uno o varios encuentros. Muchos de estos docentes son de EE.UU., dadas las conexiones que el director de ph15 tiene con fotógrafos de ese país, por haber estudiado allí. En tercer lugar, y más esporádicamente, el taller propone salidas conjuntas a sacar fotografías a parques, plazas y otros lugares de la ciudad.

Uno de los puntos más importantes de la estrategia pedagógica de ph15 es brindar a los jóvenes un espacio de contención y de ayuda en cuestiones de su vida cotidiana. Los docentes tratan de involucrarse y conocer aspectos de la vida personal de los jóvenes, aprovechando que ph15 es un espacio de educación no formal. En cada encuentro preguntan 
a diferentes participantes cómo se sienten, cómo les va con el colegio, cuáles son sus novedades personales, y muchas veces intervienen ayudándolos a resolver problemas de diversa complejidad (desde apoyo escolar hasta problemas de salud, sexualidad, familiares, etc.).

Los directores de ph15 reconocen que no son expertos en pedagogía ni en políticas sociales o de promoción educativa o cultural. Sin embargo, creen que el único prerrequisito para trabajar con jóvenes de sectores populares es la sinceridad para con ellos, no prometer aquello que no se puede cumplir y hacer la tarea con responsabilidad.

En ph15 es central el proceso de creación y la calidad artística de las fotografías. En el taller los docentes no trabajan contenidos sociales o educativos, ni trabajan cuestiones sociopolíticas como insumo para el trabajo fotográfico. Por el contrario, la consigna es totalmente abierta a lo que pueda dispararle a cada participante el hecho de tener una cámara en su mano y recorrer los espacios de socialización cotidianos, adentro y afuera del barrio.

A pesar de no ser ph15 un proyecto nacido como estrategia de intervención, mucho menos de promoción de la salud, los datos de esta investigación revelan que la participación en este proyecto moviliza dimensiones personales y colectivas de la vida de los jóvenes. En el próximo apartado se analizarán cuáles son los principales cambios percibidos por los jóvenes, sus docentes y algunas madres como consecuencia de la participación en ph15, y los procesos que podrían estar facilitando dichos cambios.

\section{PH15 MOVILIZA ASPECTOS PERSONALES Y COLECTIVOS: LA PERSPECTIVA DE LOS ACTORES}

A partir del análisis de las entrevistas realizadas, es posible afirmar que la mayoría de los jóvenes, docentes y madres, identificaron cambios en los participantes a partir de su participación en ph15 y que la mayoría identifica estos cambios como positivos. En este apartado se reconstruirán dichos procesos de cambio a partir de los relatos de los entrevistados y de las categorías que emergieron de los mismos.
Los jóvenes, sus madres y los docentes identificaron tanto aspectos personales como aspectos colectivos que se modificaron a partir de su participación en ph15. En ambos casos, los cambios derivan de procesos relacionales que ocurren tanto al interior del espacio del taller como afuera del mismo, y que se refuerzan mutuamente.

Esta sección estará dividida en tres. En primer lugar, se analizarán los cambios identificados como consecuencia de procesos que ocurren al interior del taller entre docentes, alumnos y algunos invitados a partir de la estrategia pedagógica que ph15 pone en juego. En segundo lugar, se indagarán los cambios que ocurren cuando los jóvenes exhiben su obra en centros culturales y galerías frecuentadas por personas que viven fuera de Ciudad Oculta. Finalmente, se sintetizará el modo en que estos procesos se influencian y refuerzan mutuamente.

\section{AL INTERIOR DEL TALLER: PROCESOS Y CAMBIOS}

Del análisis de las entrevistas es posible inferir que la participación en ph15 implica movimientos entre los jóvenes, que ocurren en diferentes etapas. En una primera instancia, los jóvenes aprenden a manejar algunos aspectos del lenguaje fotográfico. Logran así construir una mirada sobre el mundo que los circunda que hasta ese momento no se había revelado para ellos $y$, en consecuencia, ejercitan una práctica expresiva disruptiva en relación con su percepción habitual. Muchos jóvenes señalan que, a partir de su paso por ph15, recorren su barrio y los lugares de socialización cotidianos con una atención diferente, teniendo en cuenta rincones, objetos y detalles que antes no veían o en los cuales no se detenían. En este proceso, los docentes incitan a los jóvenes a hablar sobre sus fotografías y sobre aquello que sintieron al momento de hacer cada toma. A su vez, preguntan también a los jóvenes qué sensaciones o sentimientos les transmiten las fotos de sus compañeros. Así, los participantes comienzan a vincularse con otros jóvenes del barrio en un marco novedoso: a través del intercambio de ideas y percepciones en relación con sus propias vidas. Finalmente, comienza también un proceso de 
Figura 3. PROCESOS RELACIONALES ADENTRO DEL TALLER.

MOMENTO 3

- Mayores habilidades en el manejo del lenguaje fotográfico

- Mirada más entrenada, capacidad de conectar la fotografía propia y del otro con ideas, emociones, opiniones propias y ajenas.

- Fortalecimiento del trabajo grupal.

- Establecimiento de relaciones de confianza.

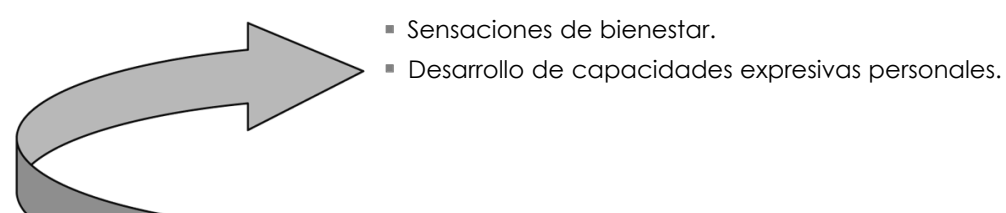

$$
\begin{aligned}
& \text { MOMENTO } 1 \\
& \text { - Manejo de algunos aspectos del len- } \\
& \text { guaje fotográfico, revelación de una } \\
& \text { mirada propia, ejercicio expresivo. } \\
& \text { - Los docentes incitan a los jóvenes a } \\
& \text { hablar sobre las fotografías y aquello } \\
& \text { que transmiten. } \\
& \text { " Espacio de encuentro entre jóvenes } \\
& \text { de distintas zonas del barrio con obje- } \\
& \text { tivos de formación y producción. } \\
& \text { - Primeros intercambios personales con } \\
& \text { los docentes. }
\end{aligned}
$$

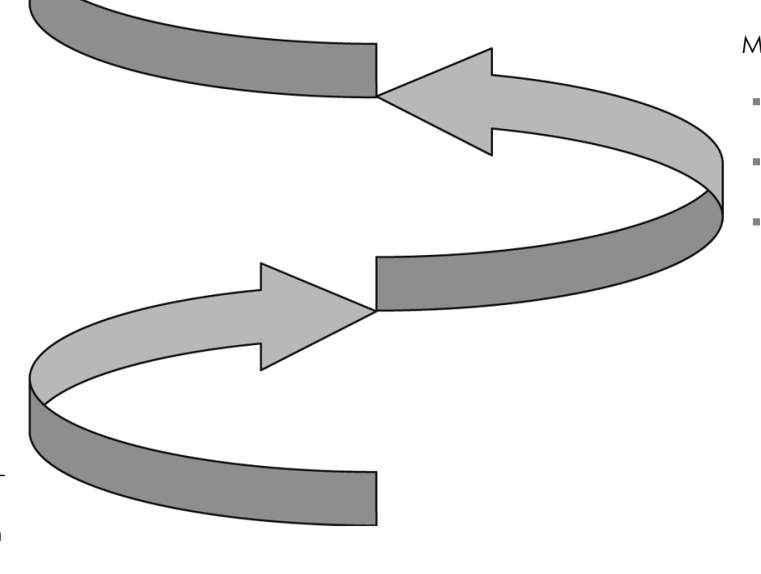

MOMENTO 2

- Producción de fotografías que consideran de calidad.

- Sensación de bienestar y orgullo por los primeros logros.

- Los jóvenes pueden vincular los resultados de algunas fotografías con aquello que los motivó a sacarlas.

Fuente: Elaboración propia

interacción con adultos, muchos de ellos muy jóvenes, que viven fuera del barrio, con quienes pueden iniciarse procesos de identificación diferentes a los que se dan con sus padres, parientes o vecinos.

Así cada joven, a su propio ritmo, va produciendo fotografías que considera buenas (por su encuadre, por aquello que expresan, por las expresiones de los rostros, por el instante que retratan, etc.) y aparece una sensación de bienestar y confianza en ellos mismos como consecuencia de estos primeros logros conseguidos. Lentamente, dependiendo también de la personalidad de cada uno, los jóvenes logran describir algo de lo que sentían cuando tomaron la foto, o qué idea les surgió al momento de hacer la toma, o simplemente, qué les llamó la atención y los incitó a sacar esa fotografía. A la vez, los participantes comienzan a interpelar las fotografías de sus compañeros en términos de sensaciones y estados de ánimo que estas fotos generan.

Con el correr del tiempo, la mayoría de los jóvenes va logrando mayores habilidades en el manejo del lenguaje fotográfico, a la vez que continúan entrenando su mirada y desarrollando la capacidad de interpelar sus propias fotos y las de sus compañeros según aquello que perciben al mirarlas. En algunos casos, los participantes logran establecer conexiones entre las impresiones que tuvieron al tomar la foto y aquello que finalmente retrataron. El trabajo de cada joven se interrelaciona, con el correr del tiempo, cada vez más con el de sus compañeros en salidas, preparación de muestras y actividades especiales. La relación entre los jóvenes y los docentes se torna más cercana y comienzan vínculos de confianza donde los jóvenes pueden hablar con ellos de sus cuestiones personales.

Estos procesos van ocurriendo a modo de espiral, y a medida que pasa el tiempo los jóvenes perciben un involucramiento con un espacio que tiene reglas, valores y actividades diferentes a las que se dan en sus espacios de socialización cotidianos. La Figura 3 esquematiza este proceso. 
Como consecuencia de los procesos relacionales que se dan en ph15, la mayoría de los jóvenes, docentes y madres identificó, por un lado, la aparición de sensaciones de bienestar y, por el otro, elementos que indicarían un desarrollo de capacidades personales.

Dentro de las sensaciones de bienestar aparecieron:

$\rightarrow$ La valorización de su propia obra y, en consecuencia, orgullo por las fotografías conseguidas.

$\rightarrow$ Mayor confianza en sí mismos y en lo que pueden producir.

$\rightarrow$ Como consecuencia de lo anterior, confianza para hablar de su trabajo fotográfico con sus compañeros y con adultos.

$\rightarrow$ Sentirse escuchados y contenidos.

$\rightarrow$ Momentos de olvido y distensión de los "problemas" cotidianos que viven en sus hogares o en el barrio.

$\rightarrow$ El sentido de pertenencia a un espacio de aprendizaje y producción artística, dejando por un rato la calle o la esquina.

Dentro de las capacidades personales mencionaron:

$\rightarrow$ Que los jóvenes hablan con más fluidez y mayor vocabulario, entre ellos y, sobre todo, con adultos (docentes, visitantes, etc.), cosa que antes no ocurría.

$\rightarrow$ Que algunos jóvenes han logrado expresar emociones, sentimientos y opiniones con mayor soltura frente al grupo.

$\rightarrow$ Los mismos jóvenes reconocieron que en ph15 aprenden "otra manera de pensar", que "madurás" o "te enseñan a sentir".

$\rightarrow$ Que están motivados y comprometidos con el espacio.

$\rightarrow$ Que adquirieron habilidades fotográficas y expresivas no solo para hacer sus fotos sino para mirar críticamente la obra de otros fotógrafos y artistas.

$\rightarrow$ Que experimentan su vida cotidiana en el barrio de manera diferente, a través de la cámara de fotos. Así, caminan siempre buscando personas o situaciones para fotografiar.

Además de estos cambios identificados con la esfera de lo personal, muchos entrevistados mencionaron que hubo cambios en las relaciones entre los jóvenes que participan del taller. En primer lugar, dicen que adquirieron cierta conciencia de grupo y que aprendieron a compartir y generar vínculos positivos entre jóvenes que viven en sectores del barrio enemistados entre sí. Esto no es menor si se tiene en cuenta el alto grado de confrontación que existe al interior de Ciudad Oculta, como se expuso anteriormente. Segundo, algunos jóvenes mencionaron que aprendieron a estar en desacuerdo con otros sin necesidad de pelear, a intercambiar ideas sin ejercer violencia física ni simbólica. Además, la mayoría mencionó que las clases grupales de crítica son centrales para mejorar sus fotografías y que, entonces, la opinión de los compañeros sobre la propia obra es importante para mejorarla. Si bien existen tensiones o diferencias al interior del grupo, no mayores a las que existen en cualquier grupo de jóvenes, se ha logrado un espacio de trabajo grupal que se fortalece cuando trabajan en conjunto para alguna exhibición, proyecto especial o, en algunos casos, viajes al interior del país. Finalmente, ph15 es un espacio en el cual se han consolidado amistades entre jóvenes que de otro modo, por vivir en extremos opuestos y enemistados del barrio, no hubieran surgido.

Llegados a este punto, es necesario aclarar que este artículo focaliza las percepciones generales mencionadas por los entrevistados como consecuencia de participar en ph15. Ahora bien, ¿están estas tendencias influenciadas por factores tales como la edad de los jóvenes y el tiempo que hace que participan del proyecto? Si bien se han realizado un número limitado de entrevistas que no permiten generalizaciones, casi la mitad del grupo ha sido entrevistada. Los datos analizados permiten sugerir que la edad no pareciera estar influenciando la percepción o no de cambios entre los jóvenes, al menos en lo que refiere a las sensaciones de bienestar, el desarrollo de capacidades y el proceso grupal. En su lugar, el tiempo y el involucramiento con el proyecto parecieran ser variables que sí influencian estas percepciones.

Los procesos de aprendizaje y cambio identificados hasta aquí por los participantes de ph15, sus madres y sus docentes ocurren en paralelo con otros procesos que se dan cuando los 
participantes muestran su obra fuera del barrio. Estos dos grupos de procesos, relacionales por definición, se refuerzan y potencian mutuamente, como se analizará en el próximo apartado.

\section{MOSTRAR SU OBRA FUERA DEL BARRIO: TOMAR LA PALABRA}

"Eso de... bueno, de tener artistas marginados y presentar obras en lugares recajetillas, reformales de la ciudad de Buenos Aires es lo más, ¿me entendés? Es decir, ese... ese pibito que está ahí, es de la villa y mirá la foto que tiene, y viene un chabón más profesional y lo mira de acá, viste, siempre de abajo [...] eso es provocar." (Participante varón, 25 años)

La exhibición de las fotografías de los jóvenes, en particular en centros culturales o galerías visitadas por sectores socioeconómicos medios y medios altos de la sociedad, ha sido mencionada por casi todos los entrevistados como una actividad central, altamente valorada del taller ph15. Sin embargo, el contacto e intercambio entre los jóvenes participantes y aquellos que visitan sus exposiciones ocurre no sin contradicciones, producto de procesos sociales de discriminación y marginalización.

En primer lugar, es importante aclarar que solo algunos adolescentes y jóvenes reconocieron haber vivido situaciones de discriminación en sus vidas y pocos dijeron haberse sentido marginados. Estos últimos han sido los entrevistados más grandes, quienes vincularon las dificultades de conseguir trabajo con el hecho de vivir en la villa. La mayoría de entrevistados más jóvenes, en particular aquellos que salen muy poco del barrio, no lograron vincular el concepto de marginalidad o discriminación con su experiencia cotidiana.

Sin embargo, a pesar de esta heterogeneidad de percepciones, que depende de la edad de los participantes y del hecho de salir mucho o poco de la villa, casi todos mencionaron que exhibir sus fotografías fuera del barrio es una de las actividades que más valoran. Es importante destacar que muchas veces estas exposiciones son cubiertas por la prensa y que han sido una de las vidrieras más importantes para que ph15 sea hoy reconocido por diversos grupos, incluidos funcionarios y técnicos del gobierno. Si bien todos los jóvenes mencionaron sentirse orgullosos de sus logros cuando exponen su obra, solo algunos interpretaron esta práctica como una forma de resistir a los discursos dominantes sobre los habitantes de la villa en general y sobre los jóvenes en particular. Estos discursos, que circulan a diario en los medios masivos de comunicación y están instalados en la opinión pública, vinculan de manera directa la pobreza con el delito, el tráfico y consumo de drogas y la violencia física y verbal $(15,16)$.

Cabe aclarar aquí que los jóvenes entrevistados, y podría arriesgarse que esto ocurre en gran medida entre jóvenes de sectores populares, no aceptan silenciosos el lugar que la opinión pública y los medios de comunicación les otorgan. Algunos manifestaron, por ejemplo, despreciar a la gente de sectores medios-altos por considerarlos demasiado superficiales, hedonistas, preocupados solo por lo que ocurre en su pequeño mundo y desconectados de los problemas más amplios de la sociedad.

Enmarcado en estas tensiones y contradicciones, no es sencillo analizar las implicancias del intercambio entre los jóvenes y quienes asisten a las muestras, entre ellos los medios de comunicación. Muchos entrevistados dijeron que el público debe quedar sorprendido al ver fotografías de calidad hechas por jóvenes de Ciudad Oculta. Algunos mencionaron que exhibir sus fotos es una manera de construir un discurso alternativo sobre los jóvenes pobres: los participantes sienten que a través de su obra demuestran a la sociedad que los jóvenes de una villa pueden hacer cosas positivas $y$, de este modo, contrarrestar en alguna medida los discursos dominantes sobre ellos.

La mayoría de los jóvenes dijo que el hecho de ser reconocidos por otros -especialmente gente que vive fuera de la villa- les generó una sensación de bienestar y mayor confianza en sí mismos y en su obra. Algunos hasta mencionaron que este reconocimiento los ayudó a sentir menos vergüenza de decir que viven en Ciudad Oculta. En este punto, cualquier analista podría preguntarse en qué medida estas percepciones implican resistencia o reproducción de la dominación, debido a que, pareciera, es el reconocimiento de esos 
otros el que, en última instancia, ayuda a los jóvenes a modificar las representaciones que tienen sobre sí mismos y sobre el lugar en el que viven. Sin embargo, una postura ética implica respoder a esta pregunta no solo desde las discusiones académicas sino a partir de las percepciones y discursos de los mismos jóvenes, que son quienes viven en primera persona estos procesos.

En primer lugar, la mayoría de los entrevistados evalúa la vinculación con otros sectores sociales como enriquecedora. Algunos dijeron que antes tenían una actitud algo desafiante cuando se cruzaban con gente de sectores medios y medios altos, actitud que los docentes interpretaron como una forma de autodefensa ante posibles situaciones de discriminación. Algunos jóvenes -en particular los más grandes y los que están hace más tiempo participando de ph15- dijeron que el taller los ayudó a construir puentes con personas que viven fuera de la villa, y a darse cuenta que estas personas no siempre son superficiales o "caretas". Un par de "graduados" dijo también que, por ejemplo, los vínculos que lograron construir con fotógrafos profesionales -a partir de las visitas del ph15 a sus muestras, de las visitas de los fotógrafos al espacio del taller y de la presencia de los fotógrafos en las muestras de ph15- les fueron muy útiles para conseguir recursos específicos (un estudio, luces) en momentos en que los necesitaron.

Sin embargo, y en paralelo a estas visiones optimistas, dos de los entrevistados trajeron a colación una pregunta que ilustra la complejidad y ambigüedad de estos procesos de diálogo entre grupos y sectores sociales que, en la Argentina de principios de siglo $\mathrm{XXI}$, comparten muy pocos o ningún espacio de socialización (17). Los dos se preguntaron si la obra de ph15 es celebrada por el público y por los medios, porque es fotografía artística de calidad o porque son jóvenes pobres haciendo fotografía, reforzando una perspectiva paternalista y etnocéntrica de celebración de aquello que los sectores populares hacen a imagen y semejanza de los dominantes.

Responder esta pregunta excede por mucho los límites de este trabajo. Para hacerlo, sería necesario complementar este estudio con una investigación de los procesos de recepción y reconocimiento que tienen lugar en las exhibiciones de ph15, o en las muestras de alguna otra expe- riencia de educación artística en barrios o villas. Sin embargo, es fundamental que ésta y otras preguntas que evidencian las contradicciones, ambigüedades y tensiones que implican estas prácticas de intervención -en este caso echando mano de prácticas de la llamada "alta cultura"- queden planteadas para futuros abordajes.

\section{ADENTRO Y AFUERA DEL TALLER: UN PROCESO DE MOVILIZACIÓN INTEGRAL QUE SE RETROALIMENTA}

Hasta aquí se han analizado dos grupos de procesos relacionales, aquellos que ocurren al interior del espacio del taller y aquellos que ocurren fuera del mismo, en particular cuando los jóvenes exhiben sus fotografías fuera del barrio.

Estos procesos no ocurren por separado sino que se refuerzan e influyen mutuamente, en lo que se describe como una segunda espiral de procesos relacionales internos y externos vinculados a la enseñanza de fotografía en un barrio popular. Si bien, como hemos visto, estos procesos implican tensiones y contradicciones, en esta sección del trabajo se abordan los mismos a partir de la problemática que pretende iluminar este artículo: la posibilidad de realizar intervenciones artístico-culturales en tanto promotoras de la salud integral y el bienestar de jóvenes en situación de vulnerabilidad social.

La Figura 4 esquematiza estos procesos, teniendo en cuenta las dos dimensiones analizadas hasta aquí.

En términos generales, entonces, a medida que pasa el tiempo, la mayoría de los entrevistados percibe un proceso de cambio, en el cual aspectos personales y colectivos se modifican. La edad no pareciera influenciar la percepción o no de cambios, siendo el tiempo y el involucramiento con el proyecto variables que sí parecieran mediar en la experimentación y percepción de los mismos.

En el próximo apartado se volverá sobre el problema que motivó este estudio: las contradicciones entre el discurso en promoción de la salud y las prácticas que bajo esa etiqueta tienen lugar. El análisis de ph15 realizado hasta el momento será conectado con el campo de la promoción de la salud. 


\section{PROMOCIÓN DE LA SALUD Y ARTE COMUNITARIO: ESTABLECIENDO CONEXIONES}

En la introducción de este trabajo se expuso brevemente el problema en el cual se enmarca esta investigación: una contradicción entre la retórica en promoción de la salud, que pregona la intervención transdisciplinaria e intersectorial para lograr el bienestar de las personas en sociedad, y las prácticas de los principales programas e iniciativas en este campo, preocupados por ampliar el acceso a los servicios de salud y por mejorar indicadores de morbimortalidad a través de la modificación de cuestiones ambientales y de comportamiento.

Teniendo en cuenta los problemas que estos enfoques generan, en particular para involucrar a jóvenes que viven en contextos de vulnerabilidad social y pobreza estructural, es pertinente introducir las siguientes preguntas: $i \mathrm{Es}$ posible interpretar una experiencia de arte comunitario como ph15 en tanto iniciativa promotora de la salud integral? Si los jóvenes, sus madres y docentes describieron procesos de cambio que los participantes de ph15 experimentaron, tanto a nivel personal como en su relación con los demás, ¿es posible leer estos procesos como cambios en dimensiones de salud en sentido amplio? Y si así fuera, ¿no estarían estas experiencias más cerca de la filosofía en promoción de la salud que la mayoría de intervenciones que bajo este rótulo se realizan? ¿Podría entonces el sector salud trabajar de manera coordinada con educación, cultura, etc., para fortalecer, replicar y extender experiencias de este tipo? Si, como sugiere Ricardo Santillán Quemes (18) la democracia cultural implica "promover situaciones de encuentro entre cotidianeidades diversas", ¿es posible pensar estas experiencias como intentos de aportar a esta democratización? Y si así fuera, ¿se borran los límites entre intervenciones de salud integral, de educación no formal, de promoción cultural, etc.?

En primer lugar, este trabajo no desconoce que la utilización del concepto amplio de salud es problemática. Si la salud implica procesos sociales, económicos, culturales y políticos (7) así como el bienestar físico y psíquico de las personas y grupos (1), todo entraría dentro de su rango de acción (19). Sin embargo, coincidimos con Helena Restrepo (5) cuando afirma que esta crítica es un sofisma, ya que, justamente, lo que el nuevo concepto de salud reconoce es que el bienestar se logra mediante la acción coordinada de diferentes sectores, instituciones y grupos. Si el sector salud acapara el conjunto de acciones de promoción de la salud, no se estaría reconociendo el aporte de otras áreas en la promoción de sociedades más saludables.

En este punto es preciso volver a la base conceptual que dio lugar al desarrollo del campo de la promoción de la salud. Esta base la constituyen los documentos que se han redactado en una serie de conferencias internacionales, organizadas bajo el ala de la Unión Internacional para la Educación y la Promoción de la Salud.

El documento fundacional y marco de referencia es la Carta de Ottawa, aprobada en Canadá en el año 1986. La Carta se apropia del concepto amplio de salud y focaliza en la necesidad de ampliar los objetivos de la promoción de la salud al trabajo con comunidades y organizaciones. Identifica cinco estrategias para la acción: construir políticas públicas saludables, crear entornos favorables, fortalecer estrategias de acción comunitaria, desarrollar aptitudes personales y reorientar los servicios de salud (20).

Si tomamos la retórica en promoción de la salud nombrada hasta aquí tendremos que, en primer lugar, la salud es un proceso colectivo con el cual se interrelacionan procesos económicos, sociales, políticos y culturales. Y que, entre las cinco estrategias de acción señaladas en un principio para promoverla, están aquellas centradas en la comunidad, particularmente aquellas que se proponen desarrollar aptitudes personales.

Como hemos visto a lo largo de este trabajo, los cambios percibidos por los jóvenes de ph15, sus madres y docentes como consecuencia de la participación en el taller de fotografía, están vinculados a sensaciones de bienestar, al desarrollo de capacidades personales y al fortalecimiento de relaciones grupales. En una primera lectura, no parecieran demasiado alejados de aquellos que las estrategias de la Carta de Ottawa promoverían.

El segundo gran documento del campo, la Declaración de Yakarta (21) no se aleja demasiado de las estrategias planteadas en Ottawa pero sí 


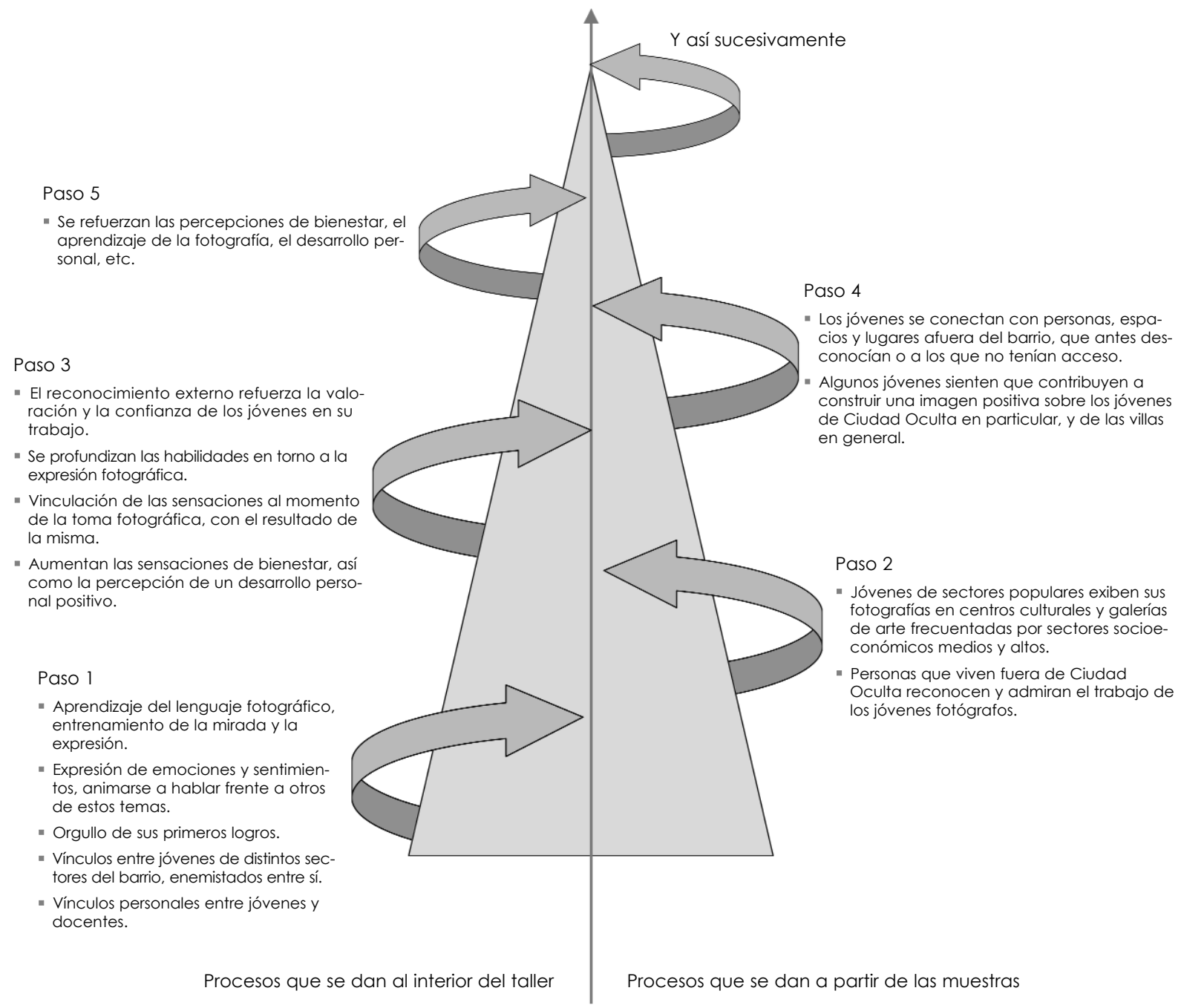

Fuente: Elaboración propia.

las focaliza desde y hacia el sector salud. Si bien en Yakarta se planteó la necesidad de avanzar en la lucha contra la pobreza y otros determinantes de la salud en países en desarrollo desde abordajes multisectoriales, se priorizaron los servicios de salud y la promoción de contenidos vinculados a la salud en sentido estricto $(2,20)$.

Envueltas en este mar discursivo, las intervenciones en promoción de la salud no han sido uniformes en los diferentes países ni al interior de los países. Y la discusión acerca de la amplitud y la especificidad de la promoción de la salud continúa abierta. Coincidimos nuevamente con Helena Restrepo (5) cuando afirma que esta discusión no se puede silenciar con una metaconceptualización de la promoción de la salud que incluya una gama de acciones focalizadas en el individuo o en el ambiente a través de las cuales, en última instancia, se mejorarían los niveles de salud o de bienestar. Algunos investigadores han 
decidido salir de este dilema teórico-metodológico identificando indicadores intermedios de salud, como ser autoestima, autovaloración, habilidades discursivas, habilidades emocionales, aptitudes para la socialización, tener y sentir un espacio comunitario como propio, por citar solo algunos $(6,22-28)$

Sin embargo, este tipo de soluciones de compromiso implican, nuevamente, el triunfo de un paradigma positivista en la investigación social en salud. Y que lo que hace falta es conocer los procesos que, desde la perspectiva de los participantes, colaboran con el mejoramiento de diversos aspectos de sus vidas cotidianas y de su relación con otros. Este trabajo se ha propuesto recuperar algunos de estos procesos experimentados por los participantes de una iniciativa de educación artística. Sus narrativas arrojan luz a la hora de pensar posibles intervenciones en promoción de la salud.

Si los enfoques de prevención/promoción centrados en el sector salud producen contradicciones, especialmente entre jóvenes que viven en contextos de vulnerabilidad, violencia y pobreza, como se expuso en la introducción de este trabajo, ¿qué elementos aporta este estudio?

En primer lugar, que si jóvenes de sectores populares perciben que un proyecto o iniciativa colabora en la generación de diversas sensaciones de bienestar a la vez que los ayuda a desarrollar habilidades personales y grupales (ya sean artísticas, creativas, expresivas o de cualquier índole), pareciera a todas luces ser un proyecto "saludable". En segundo lugar, que las metodologías de intervención, las estrategias pedagógicas y los espacios que cada proyecto construye son tanto o más importantes que el aprendizaje de una disciplina artística a la hora de conseguir los resultados narrados por los participantes. En tercer lugar, que un taller artístico expresivo permite a los participantes desarrollar productos que son valorizados socialmente, y así mostrarlos y compartirlos con otros de su entorno o externos a él. En cuarto lugar, que el hecho de mostrar su obra -en especial si su calidad es reconocida en el campo artístico- es central para la construcción identitaria de los participantes, en especial si son jóvenes que sufren procesos de marginación y discriminación, como es el caso de la mayoría de los jóvenes de sectores populares.
En quinto lugar, que no es necesario trabajar contenidos vinculados a la salud para conseguir resultados "saludables". Finalmente, que las intervenciones largas e integrales pueden producir cambios de un nivel de profundidad que los programas que duran dos o tres años y se orientan al cambio de comportamiento no han conseguido jamás.

Antes de finalizar, es preciso destacar que las intervenciones en promoción de la salud que retomen un concepto amplio de salud y, por lo tanto, sean fieles a la retórica del campo, serán a su vez iniciativas con fuertes componentes educativos y culturales. En consecuencia, si se gestionan, como sugiere Ricardo Santillán Güemes (18), teniendo en cuenta la promoción de "situaciones de encuentro" entre cotidianeidades diversas -las de los gestores y las de los destinatarios de esas iniciativas- y no a través de la imposición de elementos culturales y decisiones ajenas, pueden transformarse en herramientas para la democratización cultural. En esta misma línea y tomando la perspectiva de Pietro Paolo (29), si las iniciativas potencian recursos existentes y fortalecen a las personas, también son potenciales experiencias de promoción del desarrollo local.

Ahora bien, este análisis celebratorio del potencial de algunas intervenciones focalizadas en tanto promotoras de salud, desarrollo, etc. -en particular de aquellas que suponen la apropiación de herramientas expresivas por parte de jóvenes de sectores populares-, no debe hacernos olvidar que solo una política social universal y gestionada desde el Estado podrá modificar las condiciones de vida de grandes porciones de la población argentina que se encuentran hoy desplazadas del mundo del trabajo, de las escuelas, etc. Retomando a Eduardo Bustelo (30), es necesario promover la igualdad de oportunidades de "acceso a los frutos del progreso" y para eso no alcanza con implementar intervenciones focalizadas cuyo objetivo sea reducir la pobreza; hacen falta políticas sociales generales que actúen sobre la distribución del ingreso y la riqueza. Sin embargo, coincidimos con Jacinto y Bessega en que, si bien las intervenciones focalizadas son iniciativas con grandes limitaciones, en el marco de la actual situación social, política y económica de nuestro país "son ineludibles" (12). 


\section{NOTAS FINALES}

a. Actualmente ph15 ha logrado replicar la experiencia en otros dos barrios del conurbano bonaerense gracias a la obtención de un subsidio regular, con lo cual, se ha triplicado el número de docentes, operadores comunitarios y jóvenes participantes; así como se han diversificado sus actividades. Sin embargo el espíritu del proyecto, así como la metodología de intervención y la estrategia pedagógica siguen siendo las mismas que las que relata este trabajo.

\section{REFERENCIAS BIBLIOGRÁFICAS}

1. Organización Mundial de la Salud. Constitución de la Organización Mundial de la Salud. Nueva York; OMS; 1946.

2. Watson J, Platt S. Connecting policy and practice. En: Watson J, Platt S, editores. Researching Health Promotion. Londres, Nueva York: Routledge; 2000. p.1-20.

3. Labonte R. A community development approach to health promotion. Edinburgh: Health Education Board for Scotland/Research Unit in Health and Behavioural Change, University of Edinburgh; 1998.

4. Evans R, Stoddart G. Producing health, consuming health care. En: Evans R, Barer M, Marmor $\mathrm{T}$, editores. Why are some people healthy and others not: the determinants of health op populations. Nueva York: Aldine De Gruyter; 1994. p. $27-64$

5. Restrepo H, Málaga H. Promoción de la Salud: Cómo construir vida saludable. Bogotá: Editorial Médica Panamericana; 2001.

6. Health Development Agency. Art for health. A review of good practice in community-based arts projects and initiatives which impact on health and wellbeing. Londres: Health Development Agency; 2000.

7. Grimberg M. VIH/Sida y proceso salud-enfermedad-atención: construcción social y relaciones de hegemonía. En: Seminario Taller de Capacitación de Formadores. Buenos Aires: Lusida; 1998. p.24-31.

8. Paiva V. Analizando cenas e sexualidades: a promoção de saúde na perspectiva dos direitos humanos. En: Cáceres CF, Pecheny M, Frasca T, Careaga G, editores. Sexualidad, estigma y derechos humanos. Desafíos para el acceso a la salud en América Latina. Lima: FASPA/UPCH; 2006. p. 23-51.

9. Czeresnia D. El concepto de salud y la diferencia entre prevención y promoción. En: Czeresnia
D, Machado de Freitas C, organizadores. Promoción de la Salud. Conceptos, reflexiones, tendencias. Buenos Aires: Lugar Editorial; 2006. p. 47-63.

10. Wald G. Promoción de la salud hoy: entre la retórica y la práctica. En: VI Jornadas Nacionales de debate interdisciplinario de Salud y Población [CD-ROM]. Buenos Aires: Instituto de Investigaciones Gino Gemani, Facultad de Ciencias Sociales, UBA; 2005.

11. Urresti M. Paradigmas de participación juvenil: un balance histórico. En: Balardini S, compilador. La participación social y política de los jóvenes en el horizonte del nuevo siglo. Buenos Aires: CLACSO; 2000. p. 177-206.

12. Jacinto $C$, Bessega $C$. Un lugar en el mundo. Jóvenes vunerables en búsqueda de espacios de inclusión social. En: Forni F, compilador. De la exclusión a la organización. Hacia la integración de los pobres en los nuevos barrios del conurbano bonaerense. Buenos Aires: Ciccus; 2002.

13. Reguillo R. La performatividad de las culturas juveniles. [En línea] Estudios de Juventud 2004;(64):49- 56 [fecha de acceso 10 de junio de 2008]. URL disponible en: http://www.injuve. mtas.es/injuve/contenidos.downloadatt.action?id $=19534716$

14. Datos estadísticos comparados de villas, N.H.T. y barrios. [En línea]. Buenos Aires: Gobierno de la Ciudad de Buenos Aires; 2006 [fecha de acceso 7 de junio de 2006] URL disponible en: http://www.buenosaires.gov.ar/areas/ jef_gabinete/vivienda/estadisticos.pdf

15. Arfuch L. Crímenes y pecados: de los jóvenes en la crónica policial. Buenos Aires: Unicef; 1997.

16. Ford A, Longo EF. La exasperación del caso. Algunos problemas que plantea el creciente proceso de narrativización de la información de interés público. En: Ford $A$, editor. La marca de la bestia. Identificación, desigualdades e infoentretenimiento en la sociedad contemporánea. Buenos Aires: Norma; 1999. 
17. Auyero J. Introducción. Claves para pensar la marginación. En: Wacquant $L$, autor. Parias urbanos. Marginalidad en la ciudad a comienzos del milenio. Buenos Aires: Manantial; 2001. p. 9-31.

18. Santillán Güemes R. Lo cotidiano: principio y fin de la promoción cultural. En: Los derechos de la cultura. Buenos Aires: Subsecretaría de Cultura de la Provincia de Buenos Aires; 2001. p. 23-29.

19. Kelly $M$, Charlton B. The modern and postmodern in health promotion. En: Bunton $\mathrm{R}$, Nettleton S, Burrows R, editores. The Sociology of Health Promotion. Critical analices of consumption, lifestyle and risk. Londres: Routledge; 1995. p. 78-90.

20. Kornblit AL, Mendes Diz AM. Teoría y práctica en promoción de la salud: el caso del consumo abusivo de drogas. En: Kornblit AL, coordinadora. Nuevos Estudios sobre Drogadicción. Buenos Aires: Biblos; 2004. p. 111-120.

21. World Health Organization. Jakarta Declaration on Leading Health Promotion into the 21st Century. Jakarta; WHO; 1997.

22. Smith T. Common knowledge: the tyne and wear health action zone's arts and health projectinterim evaluation report. Durham: University of Durham; 2001.

23. Clift S. Guest editorial. Health Education. 2005;105(5):328-330.
24. Macnaughton J, White M, Stacy R. Researching the benefits of arts in health. Health Education. 2005;105(5):332-339.

25. White M. Arts in mental health for social inclusion - towards a framework for programme evaluation. En: Cowling J, editor. For Art's Sake? Society and the Arts in the 21 st Century. Londres: IPPR; 2004.

26. Dooris M. A qualitative review of Walsall Arts into Health Partnership. Health Education 2005;105(5): 355-373.

27. Everitt A, Hamilton R. Arts, health and community. A study of five arts in community health projects. Durham: CAHHM; 2003.

28. Angus J. A review of evaluation in community-based art for health activity in the UK. Londres: Health Development Agency; 2002.

29. Di Pietro Paolo L. Cultura y desarrollo local. En: Santillán Güemes R, Olmos HA, compiladores. Capacitar en cultura. Buenos Aires: Subsecretaría de Cultura de la Provincia de Buenos Aires; 2001. p. 49-64.

30. Bustelo E. De otra manera. Ensayos sobre política social y equidad. Rosario: Homo Sapiens; 2000. p. 279.

\section{FORMA DE CITAR}

Wald G. Promoción de la salud a través del arte: estudio de caso de un taller de fotografía en "Ciudad Oculta", la villa No 15 de la Ciudad de Buenos Aires. Salud Colectiva. 2009;5(3):345-362.

Recibido el 18 de marzo de 2009

Versión final presentada el 4 de junio de 2009

Aprobado el 1 de julio de 2009 